\title{
A Wearable Wireless Sensor Network for Indoor Smart Environment Monitoring in Safety Applications
}

\author{
Diego Antolín *, Nicolás Medrano, Belén Calvo and Francisco Pérez \\ Grupo de Diseño Electrónico (I3A), Departamento de Ingeniería Electrónica y Comunicaciones, \\ Universidad de Zaragoza, C/Pedro Cerbuna 12, Zaragoza 50009, Spain; nmedrano@unizar.es (N.M.); \\ becalvo@unizar.es (B.C.); fperezpellicena@gmail.com (F.P.) \\ * Correspondence: dantolin@unizar.es; Tel.: +34-876-55-34-27 \\ Academic Editor: Gonzalo Pajares Martinsanz \\ Received: 22 November 2016; Accepted: 8 February 2017; Published: 14 February 2017
}

\begin{abstract}
This paper presents the implementation of a wearable wireless sensor network aimed at monitoring harmful gases in industrial environments. The proposed solution is based on a customized wearable sensor node using a low-power low-rate wireless personal area network (LR-WPAN) communications protocol, which as a first approach measures $\mathrm{CO}_{2}$ concentration, and employs different low power strategies for appropriate energy handling which is essential to achieving long battery life. These wearables nodes are connected to a deployed static network and a web-based application allows data storage, remote control and monitoring of the complete network. Therefore, a complete and versatile remote web application with a locally implemented decision-making system is accomplished, which allows early detection of hazardous situations for exposed workers.
\end{abstract}

Keywords: wearable sensor node; wearable wireless sensor network (W-WSN); $\mathrm{CO}_{2}$ smart detection; safety applications; remote web application

\section{Introduction}

The growing advances in the last two decades in low-power wireless communications, the constant downsizing in electronic devices and the progressive increase of computational power in low-cost microcontrollers have fostered the emergence of cyber-physical systems (CPSs). A CPS [1,2] consists of a physical structure (a natural area, house, facility or even a human body) where a set of sensors monitors the main system parameters in order to obtain maximum knowledge and/or take advantage, with a minimum impact to the environment. Sensors interact with each other in order to obtain high-level information of the monitored physical system, to control some internal processes or to improve some characteristics, without the requirement of interaction with a central control system [3-8].

In safety monitoring, in industrial environments the usual approach lies in deploying static wireless sensor nodes in the area of interest. In this way, ambient monitoring systems based on wireless sensor networks (WSN) can be found in different scenarios such as refrigerated chambers [9], chemical production plants [10], or in modern steel mills to detect carbon monoxide [11]. In such potentially dangerous scenarios, continuous monitoring using wearable body sensor networks-added to work wear-will increase early detection of threatening situations for exposed workers. Wearable wireless sensor network (W-WSN) systems are a particular CPS case where sensors are deployed on the user clothing and/or body to monitor physiological parameters, environmental conditions, or both. Unlike conventional WSNs, W-WSNs consist of fewer and smaller nodes, covering less space. A typical wearable wireless sensor network consists of several wearable nodes connected between them or 
connected to a static WSN. Each wearable node includes low-power sensors, a wireless transceiver, electronic processing elements (microcontroller and interface systems) and the power supply unit, which must be miniaturized, lightweight and long lasting.

This paper presents a complete W-WSN system based on a custom wearable sensor node using a low-power low-rate wireless personal area network LR-WPAN communications protocol, intended for monitoring ambient parameters in potentially harsh environments where carbon dioxide $\left(\mathrm{CO}_{2}\right)$ gas leaks may occur. The proposed node contains two main sensors: $\mathrm{CO}_{2}$ concentration is measured by an IRC-A1 gas sensor from Alphasense (Essex, UK) with a measurement range from 0 to 50,000 ppm [12]; temperature and humidity are complementary parameters, measured by a SHT11 digital humidity sensor (Sensirion AG, Zurich, Switzerland) [13]. These nodes are designed to be connected to a deployed static network. A web-based application allows data storage, remote control and monitoring of the complete network, therefore achieving a complete and versatile remote web application with a locally-implemented decision-making system.

The paper is structured as follows: Section 2 provides a brief background to better situate the present work. Section 3 presents the description of the sensor nodes, including the core hardware, microcontroller programing technologies and networking. Within the implementation strategies, special attention has been paid to reach optimal energy handling, essential to achieving long battery life. Section 4 comprises experimental results. Section 5 explains the operation of the complete remote web application system and finally, Section 6 presents the conclusions and future work.

\section{Previous Work}

The proposed wearable device is based on a preliminary work [14], which served as the starting point to implement this optimized device that includes several both hardware and software improvements: an integrated battery charge system, static voltage scaling (SVS) and dynamic frequency scaling (DFS) techniques, and software routines that allow automatic node connection to a previously-deployed WSN. The $\mathrm{CO}_{2}$ sensor board includes a custom low-power astable circuit that excites the gas sensor, so the sensor response time keeps constant and independent of the microcontroller power mode. In addition, an acoustic buzzer has been included to advise the user about hazardous $\mathrm{CO}_{2}$ levels in the nearby environment.

The wireless sensor network used as a fixed infrastructure where portable sensors can be added is based on a work by Antolín et al. [15]. When deployed indoors, the power consumption requirements of this static WSN can be relaxed by using suitable medium-sized batteries or even a mains power connection, so the duty cycle and number of physical external parameters to be measured per node can be increased without jeopardizing the system operating life. Static nodes are configured as router devices, while wearable sensors are configured as end devices. This guarantees that wearable nodes are always connected to the network through static devices, which allows its physical location to allow early detection of gas leakage.

\section{Wearable Wireless Node Architecture}

\subsection{Wearable Sensor Node Hardware Design}

To achieve a truly wearable sensor device, key hardware design requirements are minimum size, efficient power management and a compact rechargeable battery system. Figure 1 shows the block diagram of the developed sensor node, and Figure 2 shows its photograph. It consists of a PIC18F26J50 8-bit microcontroller $(\mu \mathrm{C})$ (Microchip Technology Inc., Chandler, AZ, USA), with nanoWatt technology, selected for its cost-characteristics tradeoff and low power consumption. It owns several energy-saving working modes and independent clock lines that can be addressed to different peripherals according to its timing requirements. It can be dynamically powered at a voltage ranging from $2.0 \mathrm{~V}$ to $3.6 \mathrm{~V}$, with a quiescent current of $6.2 \mu \mathrm{A}$ in non-sleep modes. 


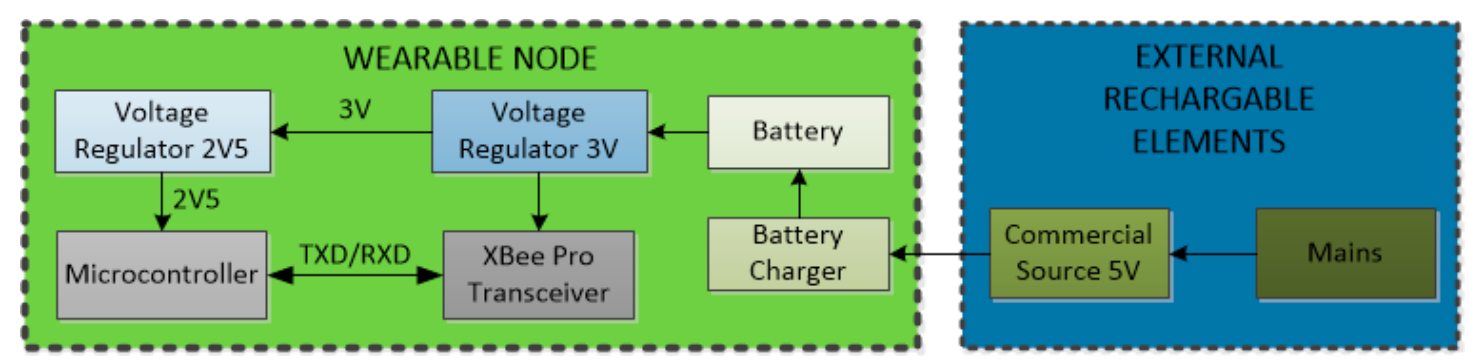

Figure 1. Wearable sensor node block diagram.

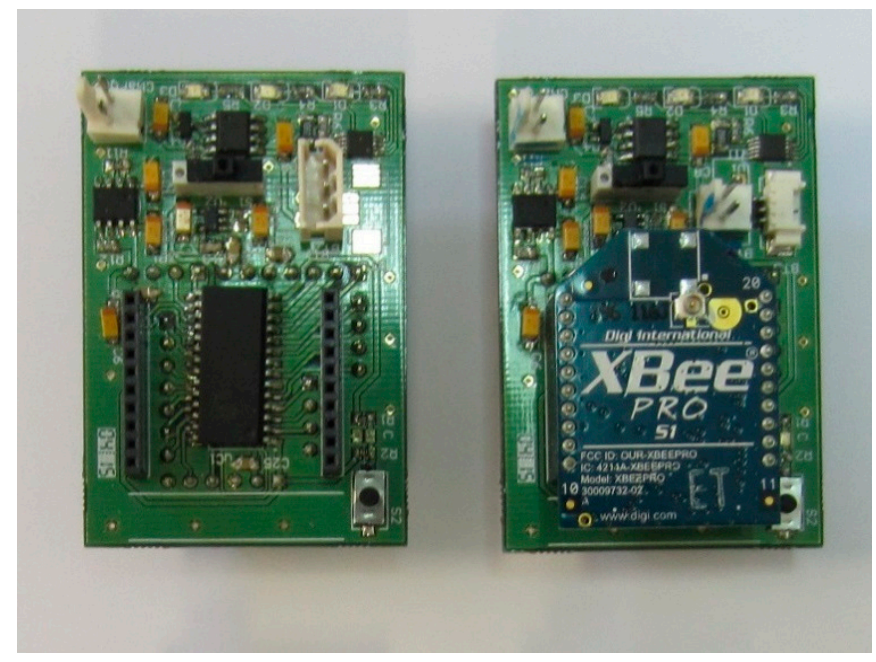

Figure 2. Wearable sensor node photograph: (Left) without XBee; (Right) with XBee.

The microcontroller manages the node operation, i.e., sensor acquisition; data collection and storage; building of the data frame to be sent by the radio frequency (RF) transceiver; sending of the data frame to the RF transceiver through an RS-232 protocol; and monitoring of the node state (energy mode, initialization, etc.). The node RF transceiver is an XBee module with a wire antenna to reduce the size. It includes the DM-24 firmware from DigiMesh that works in the $2.4 \mathrm{GHz}$ Industrial-Scientific-Medical (ISM) band with an IEEE 802.15.4 protocol running in the transceiver, making compatible the connection of this device to a deployed WSN and allowing drawing up of its communications structure.

The sensor node includes a novel and compact power supply system similar to those available in smartphones and other portable consumer electronic devices. It consists of a MCP73833 battery charger circuit from Microchip, a rechargeable battery and two low dropout (LDO) regulators to provide the power voltage levels required by the different components. The selected battery is a $3.7 \mathrm{~V}$ and $800 \mathrm{mAh}$ Lithium Polymer (LiPo) LP-573442-1S-3 that meets the W-WSN node energy requirements with a small size. The voltage regulators provide stable, well-defined voltage levels from the decreasing battery voltage: an MCP1725 LDO voltage regulator (Microchip Technology Inc., Chandler, AZ, USA) provides a $3 \mathrm{~V}$ constant voltage to power the XBee RF transceiver. The second LDO regulator (TC1015 also from Microchip) provides a constant $2.5 \mathrm{~V}$ level to power the rest of node electronics.

Sensors are housed on a specific printed circuit board (Figure 3) connected to the sensor node through a pin in-line connector. This allows an easy replacement for damaged or obsolete sensors without modifying the main node architecture. The version herein presented measures of humidity, temperature and $\mathrm{CO}_{2}$ concentration. A SHT11 (Sensirion AG, Zurich, Switzerland) provides the relative humidity and temperature information, with an operation range of $0 \%$ to $100 \%$ and $-40{ }^{\circ} \mathrm{C}$ to $124{ }^{\circ} \mathrm{C}$, respectively. The gas detection is performed by a non-dispersive infrared IRC-A1 $\mathrm{CO}_{2}$ sensor 
from Alphasense (Essex, UK) $[12,16]$ in the range of 0 to 50,000 ppm to match safety applications. This sensor requires $2 \mathrm{~Hz}$ square wave excitation, a preheating time of $30 \mathrm{~min}$ and up to $40 \mathrm{~s}$ of response time. Our conditioning circuitry has been adapted from [17], to fit the $\mu \mathrm{C}$ analog-to-digital converter (ADC) input voltage requirements.

The 2-Hz square signal required by the sensor is directly provided by an independent astable circuit based on operational amplifier MAX4038 (Maxim Integrated, San José, CA, USA) instead of a digital output from the microcontroller. Thus, the microcontroller can be set to a low-power mode, reducing the energy consumption, while the sensor keeps always active to minimize its response time.

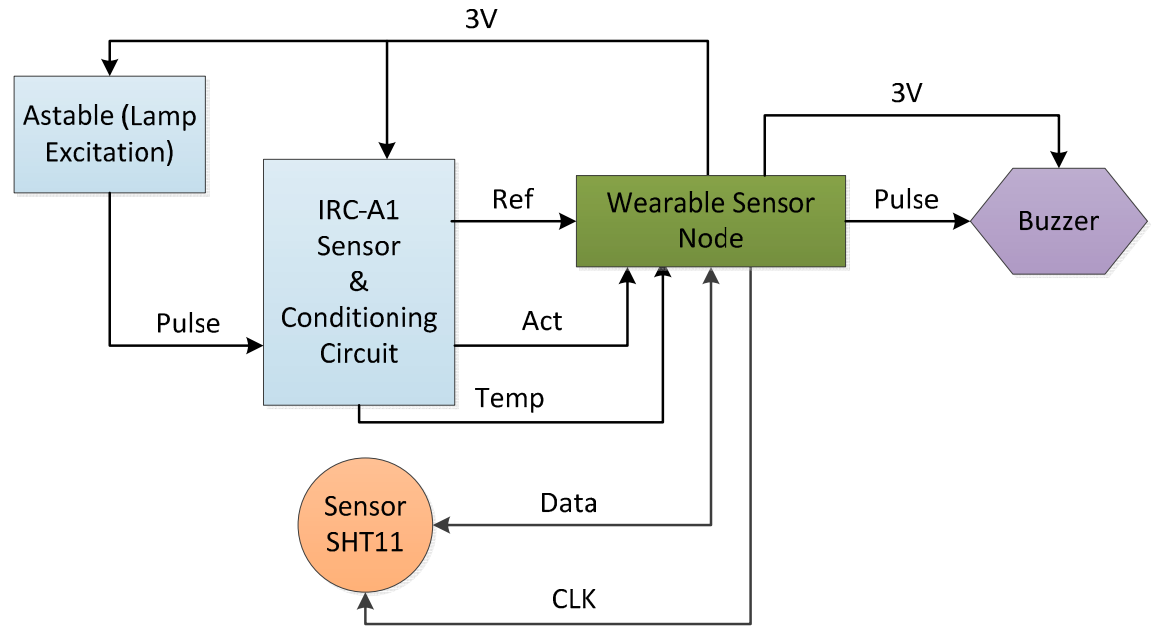

(a)

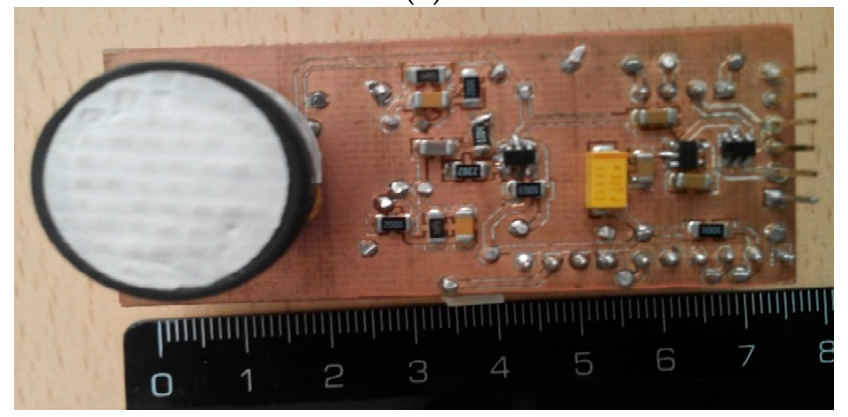

(b)

Figure 3. Sensor board and node connection: (a) block diagram; (b) photograph.

The three signals (reference, active and temperature voltages) in the IRC-A1 sensor must be used to calculate the $\mathrm{CO}_{2}$ concentration, by applying the equations given in the sensor datasheet. However, due to the complexity of the required equations and the use of a low-cost microcontroller as a sensor node manager, the microcontroller includes look-up table (LUT) data that allows for a coarse estimate of the measured $\mathrm{CO}_{2}$ levels, while an acoustic alarm using a buzzer included in the board is activated when concentration exceeds a predetermined level.

\subsection{Wearable Sensor Node Software}

Figure 4 shows the operation flowchart of the wearable sensor node software. Once the power is switched on for the very first time, the microcontroller is firstly configured using locally-stored default parameters: duty/sleep timing, operation frequencies, etc. Next, the RF transceiver is initialized following a microcontroller request, initializing the universal asynchronous receiver-transmitter (UART), configuring the interrupt service routine (ISR) and starting the wireless network joint process. Network joining requires an XBee software reset, then a commissioning command is sent to the network 
coordinator node to indicate that a new device is joining the network, and finally the transceiver is sent to sleep mode. The delays shown in the flowchart are required by the transceiver for a suitable processing of the commands.

Once both microcontroller and transceiver have been initialized, sensor input/outputs and peripherals (such as the analog-to-digital converter) are configured. Configuration is completed by enabling the microcontroller to receive interrupts from the transceiver, then driving the node to sleep mode. Once fully configured, the node operation consists of a main loop, where the microcontroller is awakened by an interrupt request from the XBee. Next, the operation frequency increases in order to reduce the acquisition time, the sensor data are collected, compared to the values stored in the LUT and sent to the network coordinator through the transceiver. Finally, the operation frequency is decreased and the system goes back to sleep mode until a new XBee interrupt is produced.

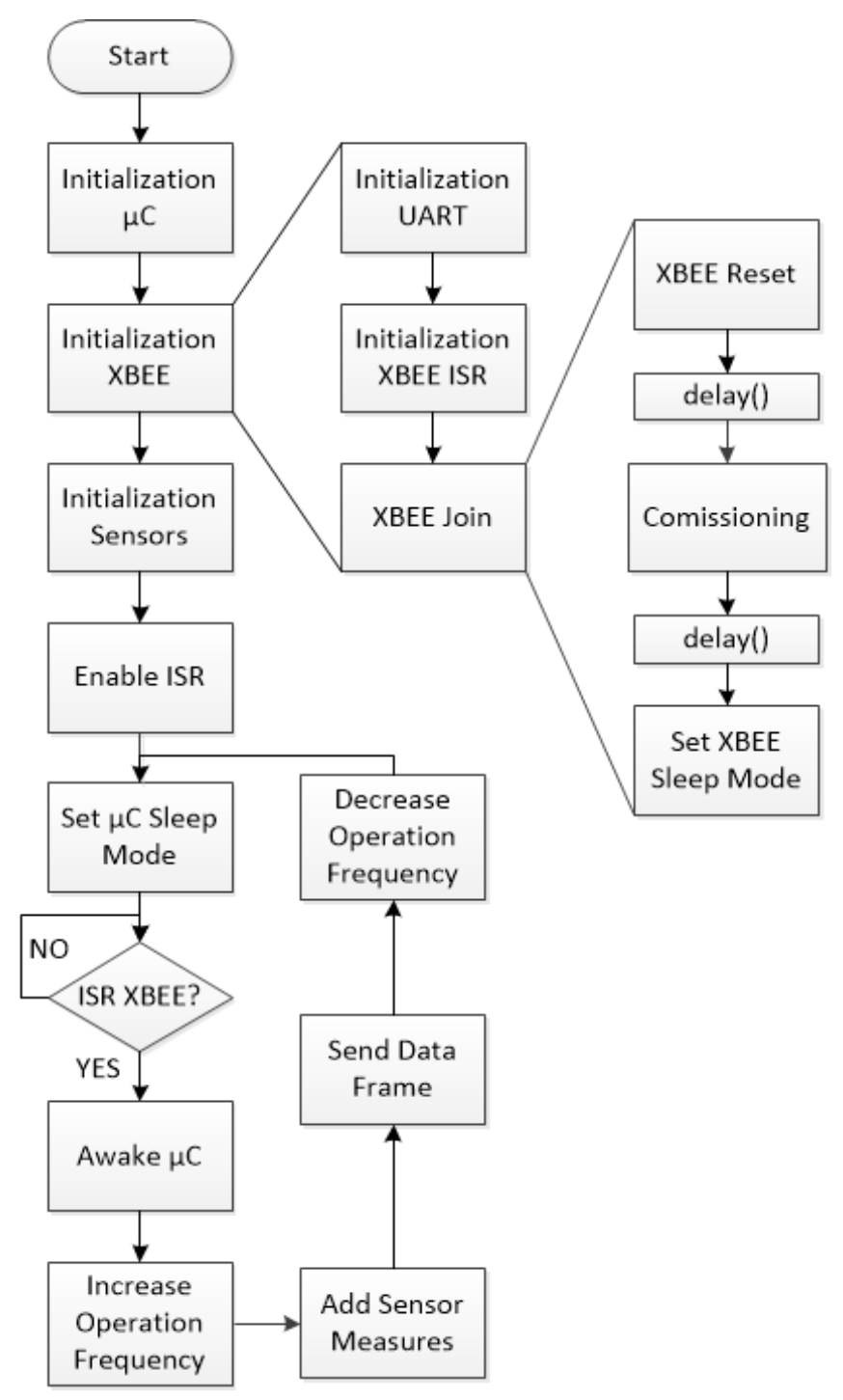

Figure 4. Flowchart of sensor node software operation. ISR: Interrupt service routine.

\section{Network Test}

The proposed system has been tested in the indoor deployment shown in Figure 5. Each room includes a static node (green), which sends ambient parameters through multi-hop transmission to the node coordinator (orange, room \#3), and then information is sent to a host device. 


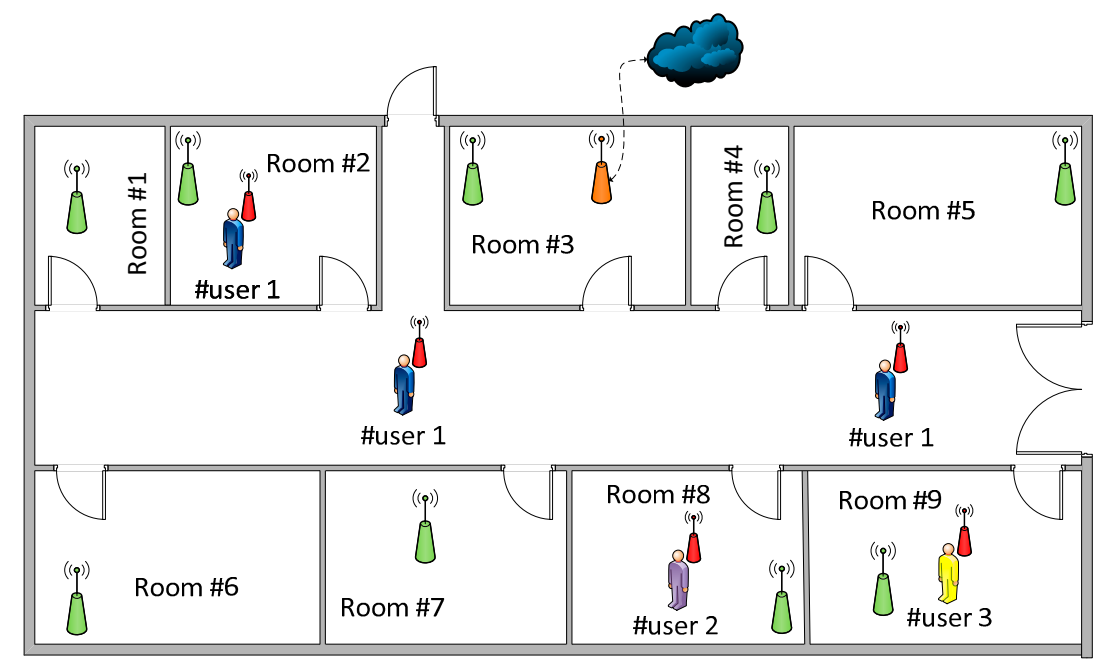

Figure 5. Indoor wearable wireless sensor network (W-WSN) deployment in the application test scenario (dimensions: $50 \mathrm{~m} \times 15 \mathrm{~m}$ ).

Wearable nodes (red, Figure 5) connect to the deployed network through the nearest fixed device, therefore providing information about the owner position and displacement. Because data transmissions are performed over short distances, RF transmission power in mobile devices is set to the minimum available value, i.e., $10 \mathrm{dBm}$ for XBee modules [18]. Node locations are upgraded every time the network nodes send data to the host system. Busy periods are configured every $15 \mathrm{~s}$, so the all the monitored parameters are sent from the wearable and static nodes via the host system to the processing application which processes the data, activating suitable protocols in case of unusual magnitude levels.

To check the correct operation of the proposed W-WSN, wearable nodes were positioned on volunteers moving along the monitored area. Figure 6 shows the measurements provided by one of the volunteers wearing a node for three consecutive days, for periods of 3.5-4.5 h. Figure 6a presents measurement results for humidity, while Figure $6 \mathrm{~b}$ shows the $\mathrm{CO}_{2}$ concentration. $\mathrm{CO}_{2}$ peaks correspond to measurements when the user entered one of the dependencies occupied by 26 students.

To validate the network link quality, the different packet error ratios (PER) have been determined. The results (Table 1) show a PER for static nodes higher than in the case of mobile nodes connected to static devices, mainly due to the fact that static links are in non-line of sight, while the mobile nodes are connected to the network with line of sight. The increase of errors in packet transmission due to node reconnections is related to changes in user location.

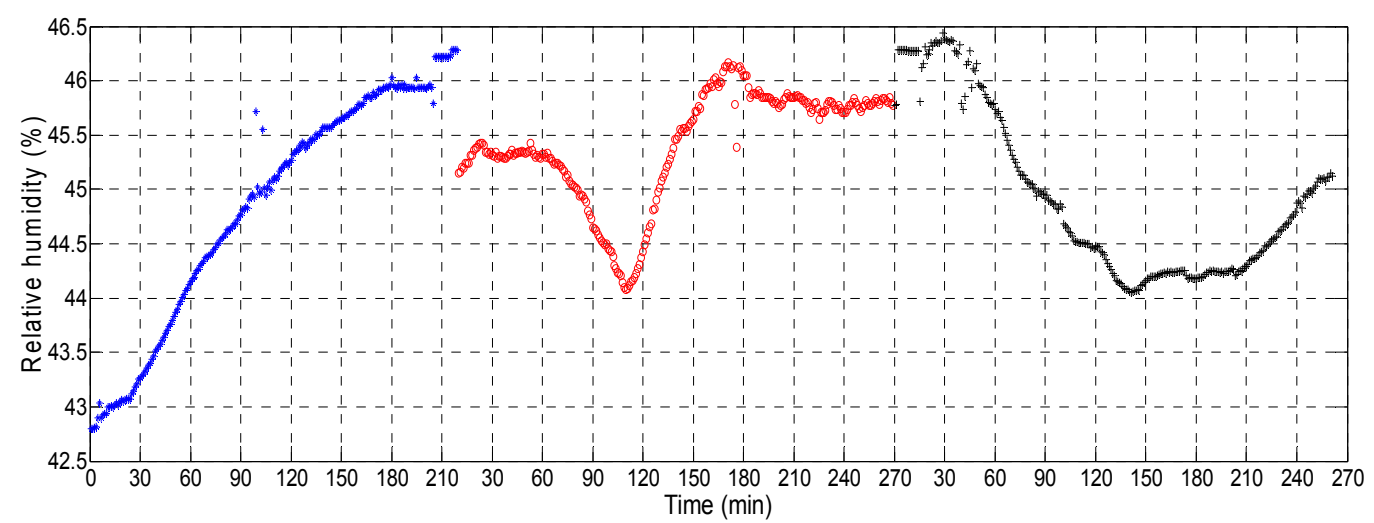

(a)

Figure 6. Cont. 


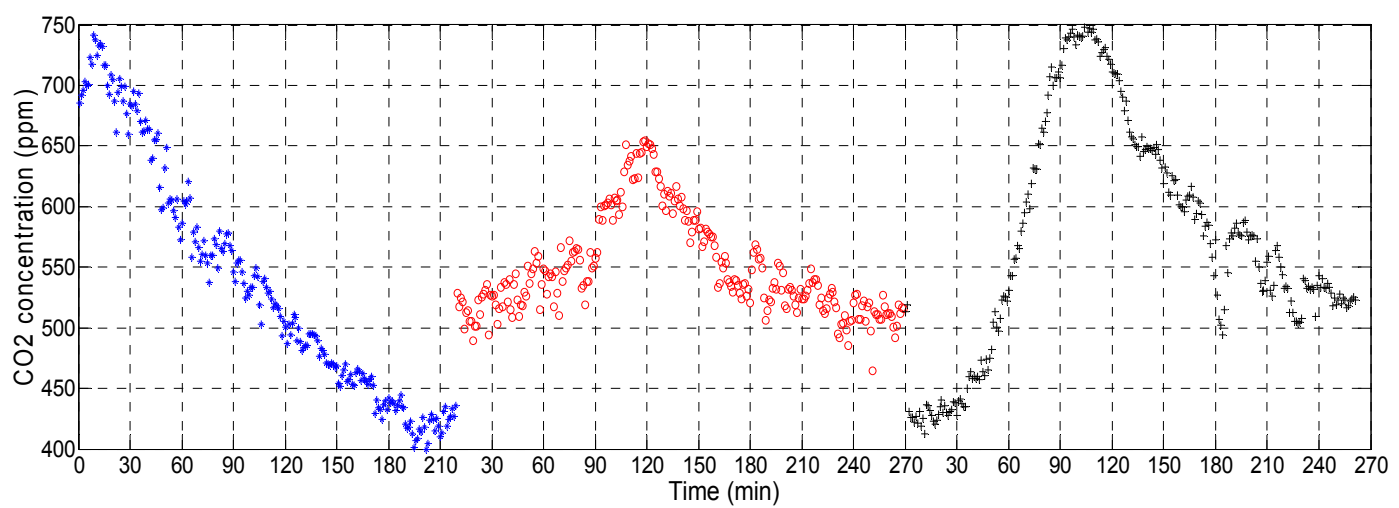

(b)

Figure 6. (a) Relative humidity acquired during laboratory sessions; (b) $\mathrm{CO}_{2}$ concentration acquired during these same periods.

Table 1. Packet Error Ratio.

\begin{tabular}{cc}
\hline Node & Rate (\%) \\
\hline Static Node & 2.6 \\
Mobile Node Connected to Static Router & 0.95 \\
Mobile Node Reconnecting to Other Router & 3.4 \\
\hline
\end{tabular}

An important issue in the design of a wearable wireless sensor node is its operating lifetime. To obtain a reliable node lifetime estimation, the evolution of the battery voltage and energy level in real operation conditions has been measured and numerically modeled. First, it was verified that the experimental power consumption is constant in each one of the node states along time. Then, to obtain the battery discharge for the $3.7 \mathrm{~V}, 800 \mathrm{mAh}$ LiPo LP-573442-1S-3 battery, the current node consumption profile was measured for several battery voltage values (Figure 7). As the figure shows, the current consumption is also independent of the battery output voltage: $5.5 \mathrm{~mA}$ in sleep mode (mainly due to keeping the $\mathrm{CO}_{2}$ sensor powered to allow fast measurement without need for warm-up time), $69 \mathrm{~mA}$ in acquisition mode and $60 \mathrm{~mA}$ in RF transmission. This is due to the use of LDO voltage regulators to power the node at fixed voltages from the battery varying voltage.

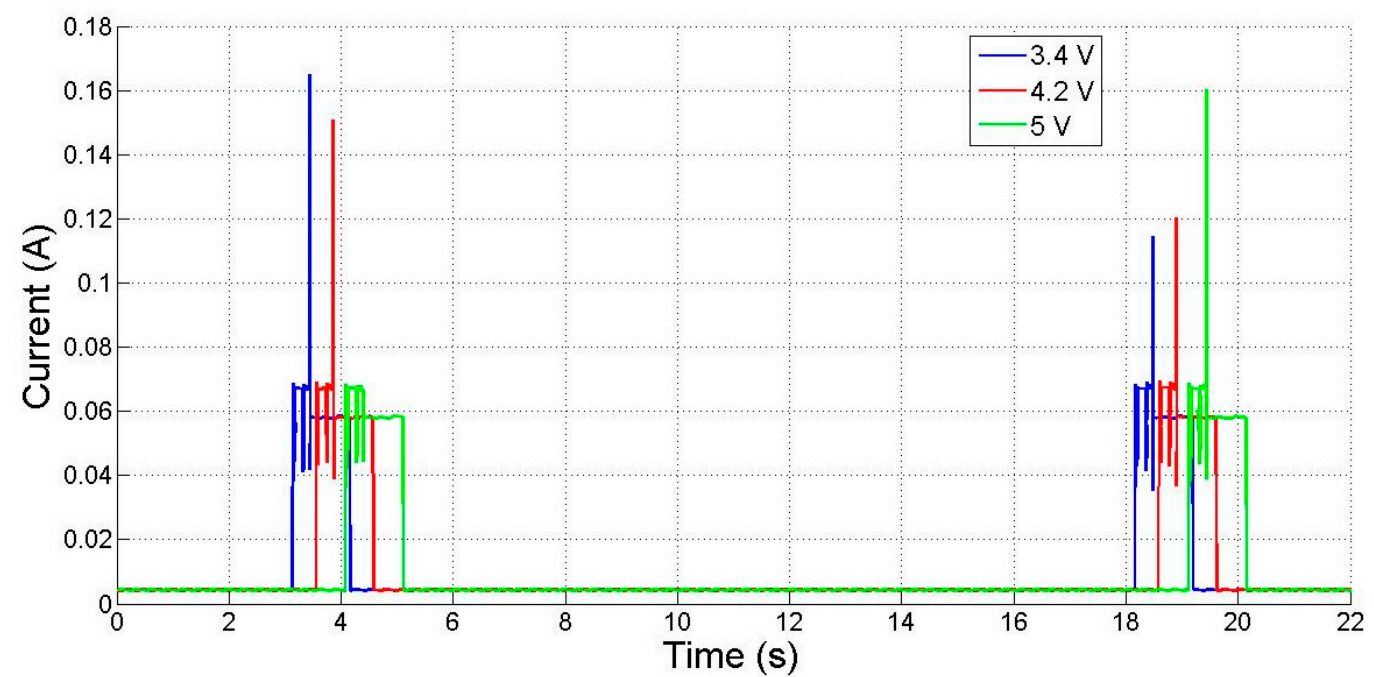

Figure 7. Mobile node current consumption. Nodes are biased by a nominal $3.7 \mathrm{~V}$ battery, charged to $5 \mathrm{~V}$ (green), $4.2 \mathrm{~V}$ (red) and $3.4 \mathrm{~V}$ (blue). 
The battery discharge at constant current is shown in Figure 8: the battery voltage drastically drops after its value falls below $3.5 \mathrm{~V}$. For the useful region, it is modeled according to

$$
\begin{aligned}
v(t)= & -1.08 \times 10^{-13} \times t^{5}+1.493 \times 10^{-10} \times t^{4}-7.36 \times 10^{-8} \times t^{3}+1.729 \times 10^{-5} \times t^{2} \\
& -3.047 \times 10^{-3} \times t+4.125(v \text { in volts, } t \text { minutes })
\end{aligned}
$$

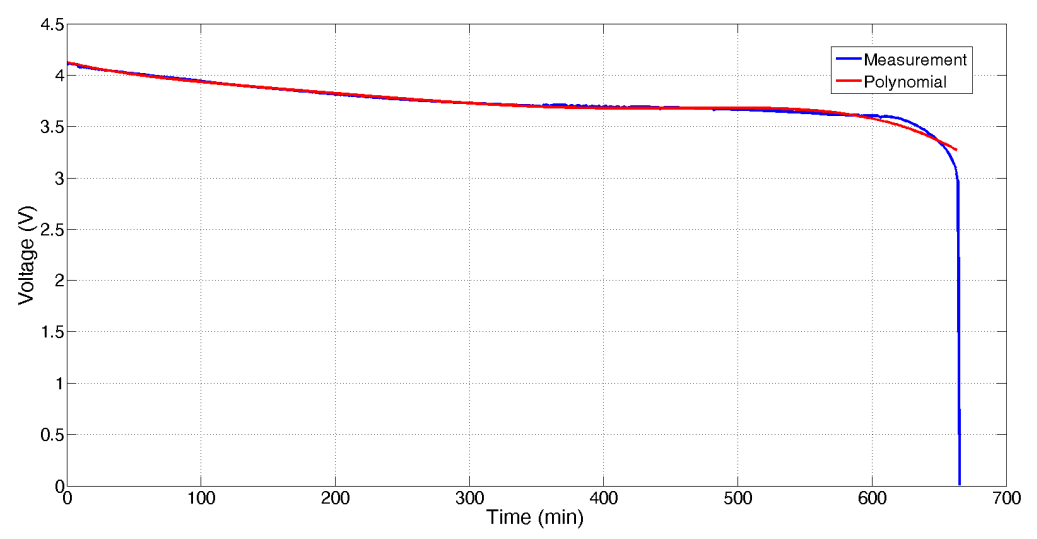

Figure 8. Experimental battery voltage (V) vs. time (min) for a constant $60 \mathrm{~mA}$ discharge.

Based on the results presented in Figure 8, the relationship between the remaining energy and the voltage provided by the battery can be obtained. The result is presented in Figure 9. This behavior is approximately given by:

$$
C(v)=-1.243 \times 10^{5} \times v^{2}+1.026 \times 10^{6} \times v-2.073 \times 10^{6}(v \text { in volts, } C \text { in mAmin })
$$

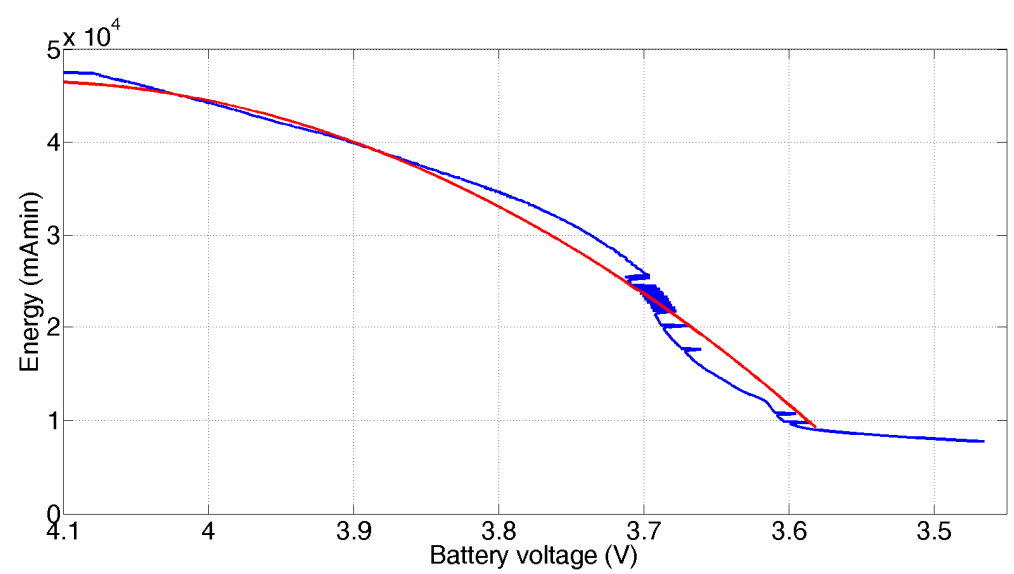

Figure 9. Experimental (blue) and polynomial (red) fit for battery discharge (mAmin) vs. battery voltage.

On the other hand, the equation of the node battery discharge in each working cycle can be obtained according to the process described in [15] as follows

$$
C=\int_{t_{0}}^{t_{1}} i_{1}(t) d t+\int_{t 1}^{t_{2}} i_{2}(t) d t+K \int_{t 2}^{t_{3}} i_{3}(t) d t
$$

where $i_{x}(t)$ represents the current in the three different node states: sleep, with time limits $t_{0}$ and $t_{1}$; measure, with time limits $t_{1}$ and $t_{2}$; and RF transmission, with time limits $t_{2}$ and $t_{3}$. Since the current consumption in each state (see Figure 7) is constant, Equation (3) can be simplified to 


$$
C=i_{1}\left(t_{1}-t_{0}\right)+i_{2}\left(t_{2}-t_{1}\right)+i_{3}\left(t_{3}-t_{2}\right)
$$

In this way, it is possible to estimate the battery discharge as a function of time as

$$
C(t)=-6.575 \times t+47998.38(C \text { in mAmin, } t \text { in minutes })
$$

Taking into account that the useful operating voltage is limited to $3.5 \mathrm{~V}$, which corresponds to a remaining energy of approximately $8000 \mathrm{mAmin}$ (Figure 9), simulation results indicate a wearable node operating life of 5.07 days (Figure 10), close to the 5.34 days experimentally determined, for a duty cycle of $73.1 \mathrm{~ms}$ and $15 \mathrm{~s}$ of working cycle. These values ensure a suitable operating life for a wearable device, allowing setting down an appropriate schedule for battery recharge processes, i.e., this operability time is long enough for a portable system to be charged on a daily basis. The rechargeable system integrated in mobile sensor node is essential for this application and it is an improvement with respect to previous work.

Finally, Table 2 summarizes the main performances of the proposed wearable node and compares them with other similar solutions.

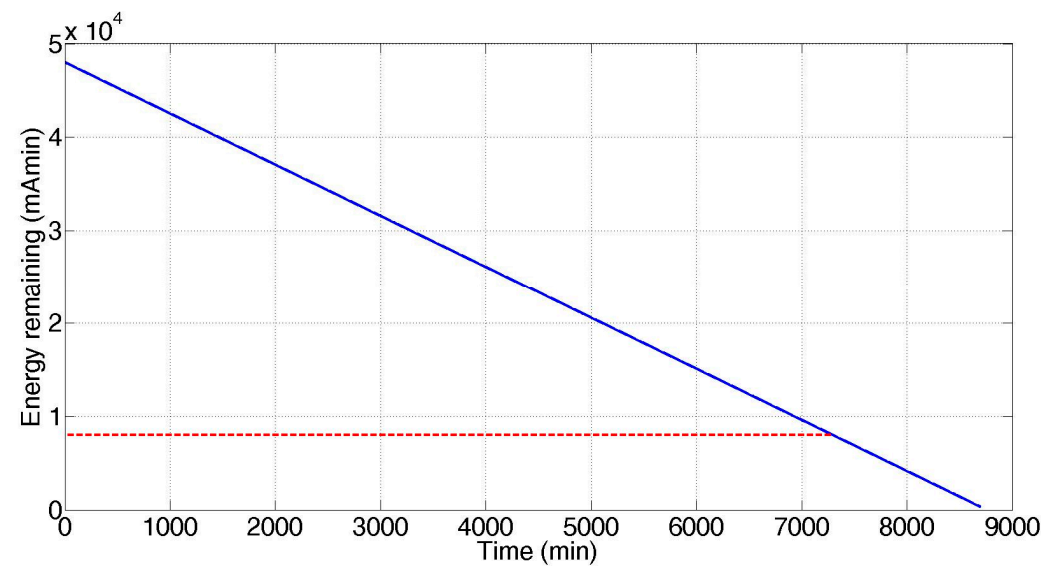

Figure 10. Lifetime estimation. Red line indicates the useful energy limit stored in the battery.

\begin{tabular}{|c|c|c|c|c|c|}
\hline Device Parameters & IRIS Crossbow & Micaz Crossbow & $\begin{array}{c}\text { TelosB } \\
\text { Crossbow }\end{array}$ & $\begin{array}{l}\text { Waspmote } \\
\text { Libelium }\end{array}$ & This Work \\
\hline \multicolumn{6}{|l|}{ Processor } \\
\hline Microcontroller & ATMega1281 & ATMega1281 & MSP430 & ATMega1281 & PIC18F26J50 \\
\hline $\mathrm{N}^{\circ}$ Bits & 8 bits & 8 bits & 16 bits & 8 bits & 8 bit \\
\hline Frequency & $\mathrm{N} / \mathrm{A}$ & $\mathrm{N} / \mathrm{A}$ & $\mathrm{N} / \mathrm{A}$ & $8 \mathrm{MHz}$ & $8 \mathrm{MHz}$ \\
\hline Active Mode Current & $8 \mathrm{~mA}$ & $8 \mathrm{~mA}$ & $1.8 \mathrm{~mA}$ & $9 \mathrm{~mA}$ & $7 \mathrm{~mA}$ \\
\hline Sleep Mode Current & $8 \mu \mathrm{A}$ & $<15 \mu \mathrm{A}$ & $5.1 \mu \mathrm{A}$ & $62 \mu \mathrm{A}$ & $<6 \mathrm{uA}^{*}$ \\
\hline \multicolumn{6}{|l|}{ RF Transceiver } \\
\hline Frequency Band & ISM $2.4 \mathrm{GHz}$ & ISM $2.4 \mathrm{GHz}$ & ISM $2.4 \mathrm{GHz}$ & ISM $2.4 \mathrm{GHz}$ & ISM $2.4 \mathrm{GHz}$ \\
\hline Outdoor Range & $>300 \mathrm{~m}$ & 75-100 m & 75-100 m & $750-1500 \mathrm{~m}$ & $750-1500 \mathrm{~m}$ \\
\hline Indoor Range & $>50 \mathrm{~m}$ & $20-30 \mathrm{~m}$ & $20-30 \mathrm{~m}$ & 60-90 m & $60-90 \mathrm{~m}$ \\
\hline Sensitivity & $-101 \mathrm{dBm}$ & $-94 \mathrm{dBm}$ & $-94 \mathrm{dBm}$ & $-100 \mathrm{dBm}$ & $-100 \mathrm{dBm}$ \\
\hline Max. Tx Power & $3 \mathrm{dBm}$ & $0 \mathrm{dBm}$ & $0 \mathrm{dBm}$ & $18 \mathrm{dBm}$ & $18 \mathrm{dBm}$ \\
\hline Receive Mode & $16 \mathrm{~mA}$ & $19.7 \mathrm{~mA}$ & $23 \mathrm{~mA}$ & $57.08 \mathrm{~mA}$ & $56.4 \mathrm{~mA}$ \\
\hline Transmission Current & $17 \mathrm{~mA}$ & $17.4 \mathrm{~mA}$ & $\mathrm{~N} / \mathrm{A}$ & $188 \mathrm{~mA}$ & $69 \mathrm{~mA}$ \\
\hline Sleep Mode & NA & $1 \mu \mathrm{A}$ & $1 \mu \mathrm{A}$ & $120 \mu \mathrm{A}$ & $<12 \mu \mathrm{A}$ \\
\hline \multicolumn{6}{|l|}{ Power Supply } \\
\hline Battery & $2 \times$ AA batteries & $2 \times$ AA batteries & $2 \times$ AA batteries & $\mathrm{N} / \mathrm{A}$ & Li-Po \\
\hline External Power & $2.7 \mathrm{~V}$ to $3.3 \mathrm{~V}$ & $2.7 \mathrm{~V}$ to $3.3 \mathrm{~V}$ & $\mathrm{~N} / \mathrm{A}$ & $3.3 \mathrm{~V}$ to $4.2 \mathrm{~V}$ & $3.5 \mathrm{~V}$ to $4.2 \mathrm{~V}$ \\
\hline
\end{tabular}

Table 2. Sensor Nodes Comparison.

${ }^{*}$ Measures without sensors, but including battery charger circuit. N/A: Not Available. 


\section{Web Data Collection and Processing}

To attain a complete and versatile system, a remote web application has been developed. A wireless sensor network is a paradigm belonging to the widest concept of Internet of Things (IoT). Therefore, proper use of the data provided by the sensors will require the application of computational methods based on cloud computing and Big Data techniques $[19,20]$. Following this new paradigm, handling large amounts of information requires the use of non-relational databases. In this work, the open-source MongoDB database [21] has been selected. MongoDB is a high performance database, with high availability and automatic information scaling stored in JavaScript Object Notation (JSON [22]) format. Therefore, it is resilient to dynamic changes in the data structure. This is an important feature because as the sensors installed in the nodes could be changed at any time, the underlying data model must be capable to support such changes in a transparent manner.

The management and monitoring of the proposed sensor system is based on a distributed architecture, with two clearly defined roles: network control; and data collection, processing and display (Figure 11).

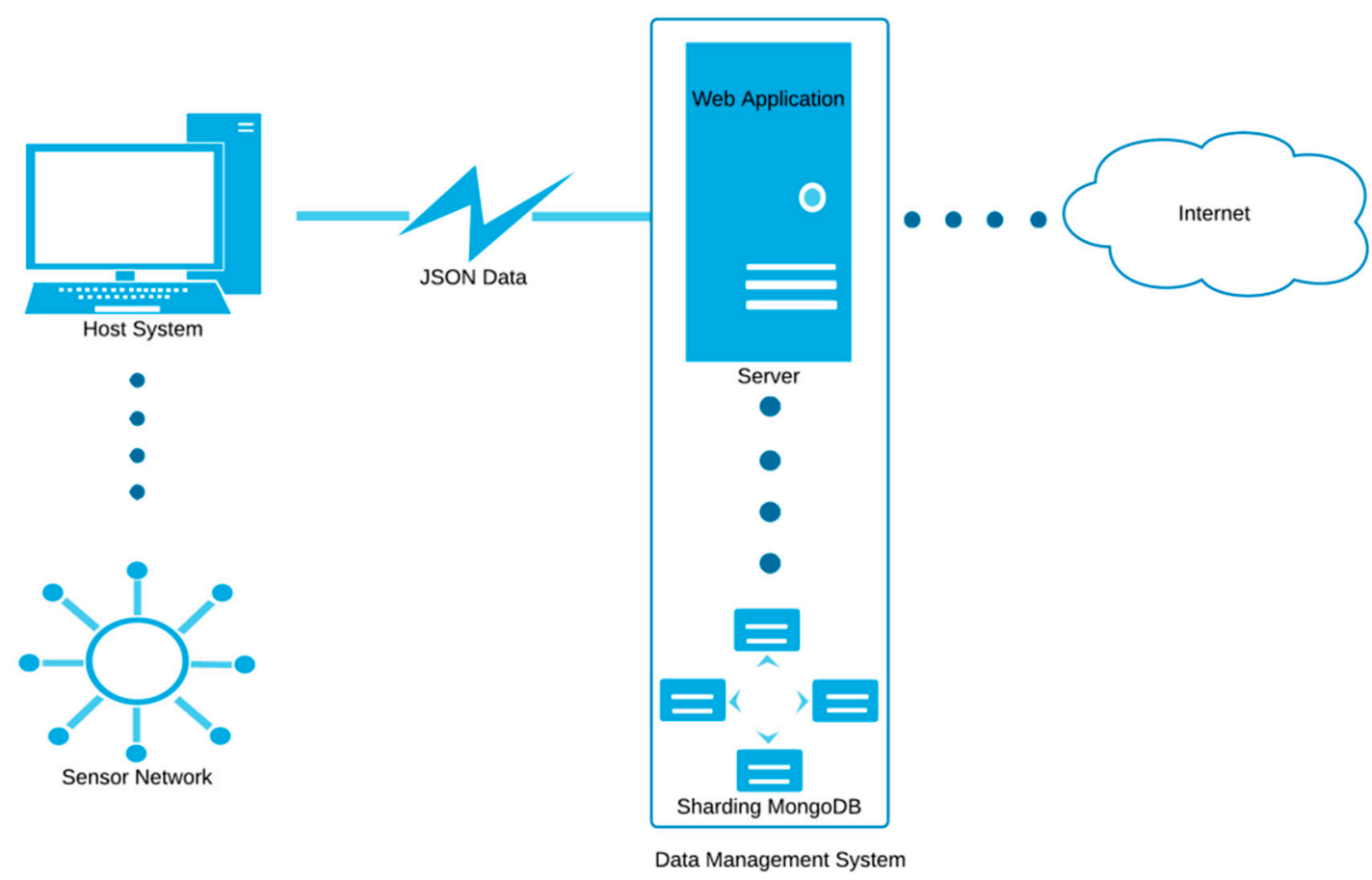

Figure 11. Interconnection of web application elements. JSON: JavaScript Object Notation.

Network control is provided by a host system connected to the sensor network through an USB-to-XBee adaptor. This host is a proxy between the sensor network and the data management system. It receives data from the network (sensor measurements and node network locations), and sends commands and parameters to the full system or to specific sensors. The host system broadcasts node discovery requests to the network periodically in order to determine in real time the mobile node location.

Commands to the sensor nodes use a high level library, abstracting the lower level details of the communication protocol (Figure 12) and encapsulating the DigiMesh frame model (Figure 13), therefore making it transparent to the end user. 

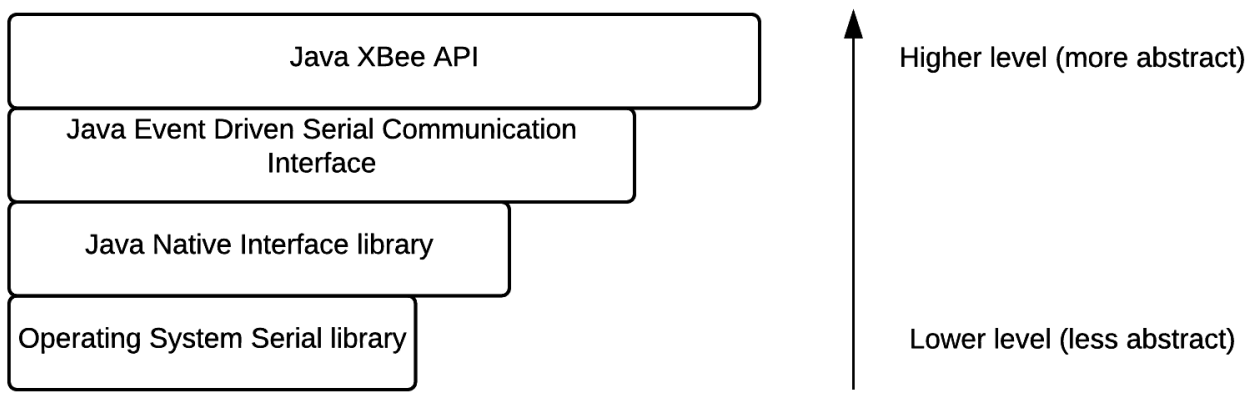

Figure 12. Abstraction layers from the operating system to the application code. API: Application program interface.

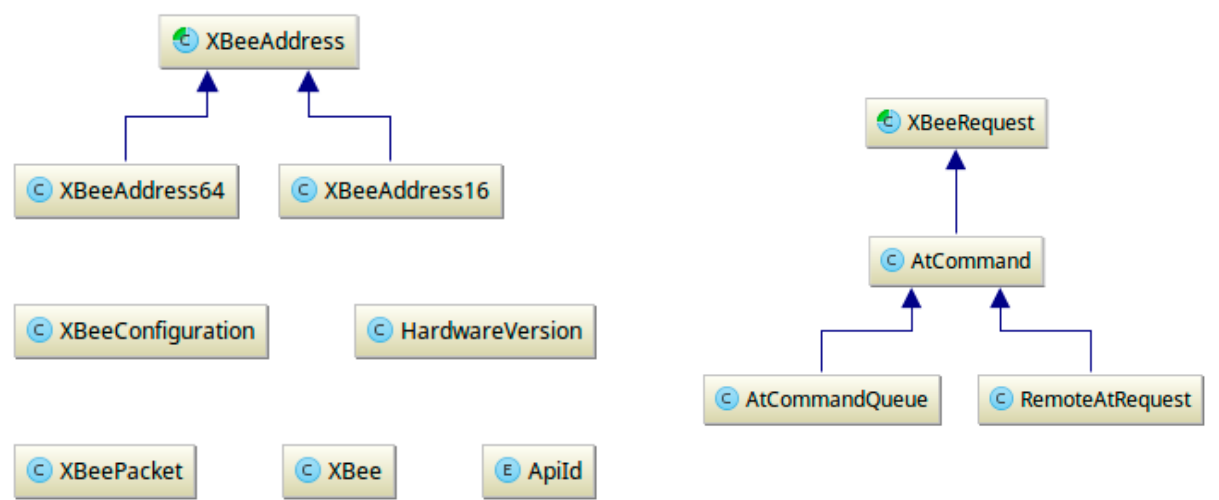

(a)

(b)

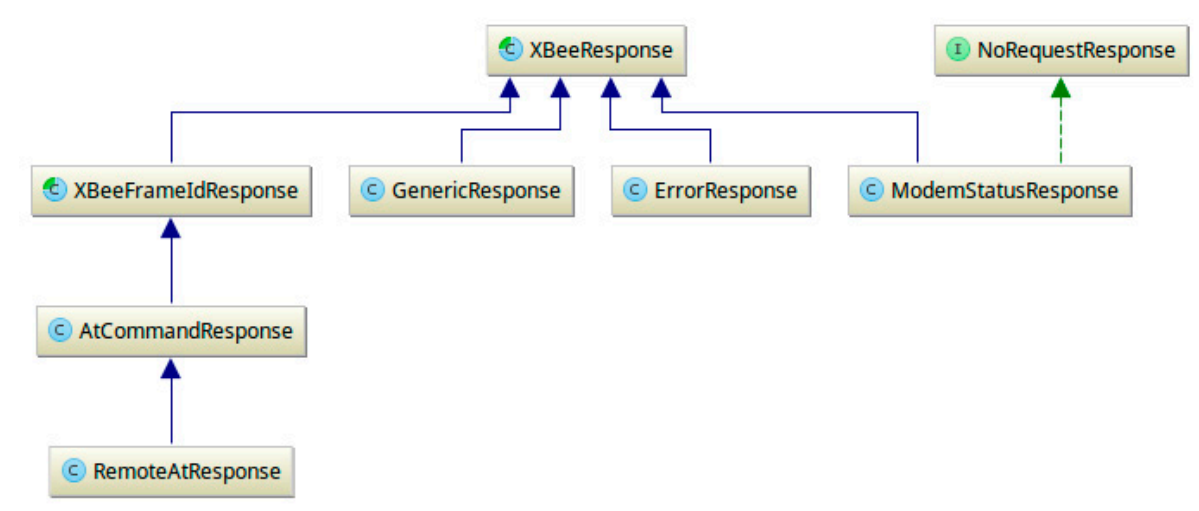

(c)

Figure 13. (a) Class hierarchy modelling the XBee according to the datasheet; (b) Class hierarchy modelling the XBee request according to the datasheet; (c) Class hierarchy modelling the XBee response according to the datasheet.

The data management system receives data from the sensor network through the host system via hypertext transfer protocol (http), formatting data in JSON. This allows sending and receiving of structured data using plain text, which simplifies and lightens the processing. JSON has many advantages over other existing formats, such as EXtensible Markup Language (XML [23]), requiring less boilerplate text to represent the data and a human-readable structure. Data management is divided in two different parts, kept in the same physical system for simplicity: the web application and the information storage system.

The web application (Figure 14) allows the user to have a graphical view of the data collected by the network, enabling dynamic monitoring of the network state. It also permits the modification of 
network parameters such as the duty cycle or the power data transmission. This developed application is based on an event-driven model, where network control tasks are executed asynchronously according to the dynamic events generated by the network, mainly the sent and received packets. The most important feature of the view layer is the ability to plot the network data gathered from the sensors. The view layer relays on an application program interface (API) that allows sending of information using JSON format, easily exportable to other systems. On the other hand, a powerful JavaScript charting library has been used to plot the network data in a timeline. The view layer code plots the measurements by sensor node using a different scale per parameter. This representation allows the user to analyze the timeline at a glance. In addition, the software is able to refresh the charts in a timely manner with the new measures generated from the nodes.

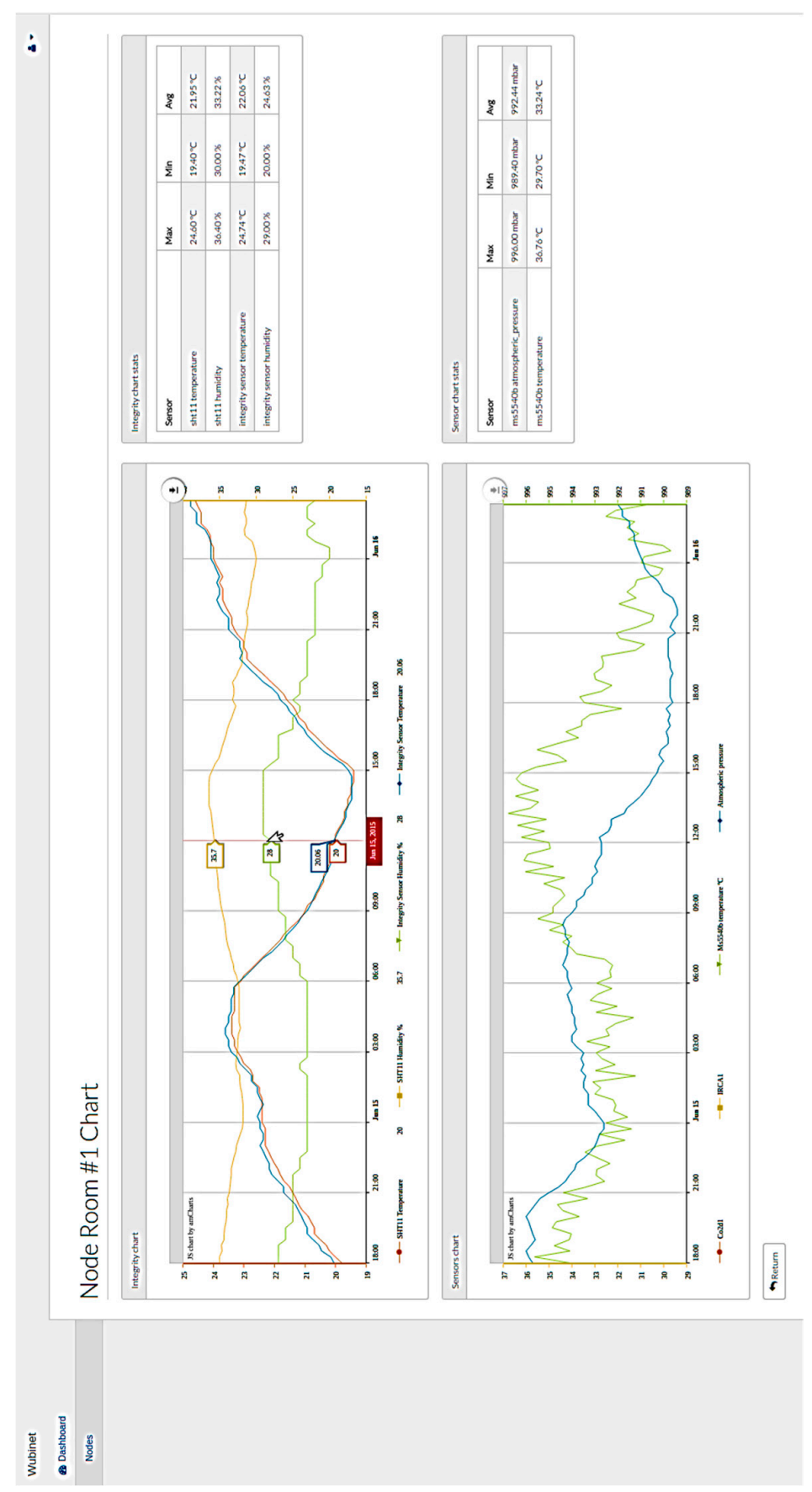

Figure 14. Web application screenshot. 


\section{Conclusions}

This work presents an indoor smart environment monitoring system for safety applications. It is based on custom wearable sensor nodes, connected to a static WSN. Through a web application the network configuration can be controlled and managed remotely, while receiving and representing the information collected by the nodes. The system has been developed for a hazardous gas environment, but could be applied to a number of other safety applications or in other areas such as the tracking of medical devices in a hospital.

Acknowledgments: This work was supported in part by TEC2015-65750-R (MINECO/FEDER, UE) and JIUZ-2016-TEC06. Authors would like to acknowledge the use of Servicio General de Apoyo a la Investigación-SAI, Universidad de Zaragoza.

Author Contributions: D.A. designed and implemented the wearable sensor node, performed its validation and the complete network test experiments. F.P. developed the web application. B.C. and N.M. supervised all the work and analyzed the results. D.A., B.C. and N.M. wrote the paper.

Conflicts of Interest: The authors declare no conflict of interest.

\section{References}

1. Lee, E.A.; Seshia, S.A. Introduction to Embedded Systems, A Cyber-Physical Systems Approach, 2nd ed.; MIT Press: Cambridge, MA, USA, 2017.

2. Marwedel, P. Embedded System Design. Embedded Systems Foundations of Cyber-Physical Systems, 2nd ed.; Springer: Dordrecht, The Netherlands, 2011.

3. Wang, H.; Liu, J.; Han, J. RS-CPS: A distributed architecture of robotic surveillance cyber-physical system in the nature environment. In Proceedings of the IEEE International Conference on Cyber Technology in Automation, Control, and Intelligent Systems (CYBER), Shenyang, China, 8-12 June 2015; pp. 1287-1292.

4. Paul, S.; Parajuli, A.; Barzegaran, M.R.; Rahman, A. Cyber physical renewable energy microgrid: A novel approach to make the power system reliable, resilient and secure. In Proceedings of the IEEE Innovative Smart Grid Technologies-Asia (ISGT-Asia), Melbourne, Australia, 28 November-1 December 2016; pp. 659-664.

5. Wu, W.; Aziz, M.K.; Huang, H.; Yu, H.; Gooi, H.B. A real-time cyber-physical energy management system for smart houses. In Proceedings of the IEEE PES Innovative Smart Grid Technologies, Perth, WA, USA, 13-16 November 2011; pp. 1-8.

6. Pang, C.; Vyatkin, V.; Mayer, H. Towards cyber-physical approach for prototyping indoor lighting automation systems. In Proceedings of the IEEE International Conference on Systems, Man, and Cybernetics (SMC), San Diego, CA, USA, 5-8 October 2014; pp. 3643-3648.

7. Dogaru, D.I.; Dumitrache, I. Cyber-physical systems in healthcare networks. In Proceedings of the 2015 E-Health and Bioengineering Conference (EHB), Iasi, Romania, 19-21 November 2015; pp. 1-4.

8. Zhang, Y.; Qiu, M.; Tsai, C.W.; Hassan, M.M.; Alamri, A. Health-CPS: Healthcare Cyber-Physical System Assisted by Cloud and Big Data. IEEE Syst. J. 2015, 99. [CrossRef]

9. Corbellini, S.; Ferraris, F.; Parvis, M.A. System for Monitoring Workers' Safety in an Unhealthy Environment by means of Wearable Sensors. In Proceedings of the Instrumentation and Measurement Technology Conference (IMTC), Victoria, BC, Canada, 12-15 May 2008; pp. 951-955.

10. Chen, L.; Yang, S.; Xi, Y. Based on ZigBee wireless sensor network the monitoring system design for chemical production process toxic and harmful gas. In Proceedings of the Computer, Mechatronics, Control and Electronic Engineering (CMCE), Changchun, China, 24-26 August 2010; pp. 425-428.

11. Jan, M.F.; Habib, Q.; Irfan, M.; Murad, M.; Yahya, K.M.; Hassan, G.M. Carbon monoxide detection and autonomous countermeasure system for a steel mill using Wireless Sensor and Actuator Network. In Proceedings of the 6th International Conference on Emerging Technologies (ICET), Islamabad, Pakistan, 18-19 October 2010; pp. 405-409.

12. Alphasense. NDIR Gas Concentration Calculation Overview. Application Note AAN 201-06. November 2014. Available online: http://www.alphasense.com/WEB1213/wp-content/uploads/2014/12/AAN_201-06.pdf (accessed on 1 February 2017). 
13. Sensirion. Sensirion SHT11 Humidity Sensor Datasheet. December 2011. Available online: https://www.sensirion.com/fileadmin/user_upload/customers/sensirion/Dokumente/2_Humidity_ Sensors/Sensirion_Humidity_Sensors_SHT1x_Datasheet_V5.pdf (accessed on 1 February 2017).

14. Antolín, D.; Medrano, N.; Calvo, B.; Garcia-Romeo, D. A Wearable Wireless Sensor Node for Safety Applications. In Proceedings of the IEEE Sensors, Valencia, Spain, 2-5 November 2014.

15. Antolín, D.; Medrano, N.; Calvo, B. Reliable Lifespan Evaluation of a Remote Environment Monitoring System Based on Wireless Sensor Networks and Global System for Mobile Communications. J. Sens. 2016, 2016, 4248230. [CrossRef]

16. Hodgkinsona, J.; Smithb, R.; On Hob, W.; Saffellb, J.R.; Tatam, R.P. Non-dispersive infra-red (NDIR) measurement of carbon dioxide at $4.2 \mu \mathrm{m}$ in a compact and optically efficient sensor. Sens. Actuators B Chem. 2013, 16, 291-295.

17. Garcia-Romeo, D.; Fuentes, H.; Medrano, N.; Calvo, B.; Celma, S.; Antolín, D. An electronic interface for measuring $\mathrm{CO}_{2}$ emissions in embedded systems. In Proceedings of the International Conference on Instrumentation and Measurement Technology Conference (I2MTC), Graz, Austria, 13-16 May 2012; pp. 417-420.

18. Digi. XBee \& XBee Pro DM 2.4 GHz Datasheet. Available online: http://www.digi.com/pdf/ds_ xbeedigimesh24.pdf (accessed on 1 February 2017).

19. Hillbert, M.; López, P. The World's Technological Capacity to Store, Communicate, and Compute Information. Science 2011, 332, 60-65. [CrossRef] [PubMed]

20. Manyika, J.; Chui, M.; Brown, B.; Bughin, J.; Dobbs, R.; Roxburgh, C.; Byers, A.H. Big Data: The Next Frontier for Innovation, Competition, and Productivity. McKinsey Global Institute, 2011. Available online: http://www.mckinsey.com/business-functions/business-technology/our-insights/big-data-thenext-frontier-for-innovation (accessed on 29 September 2016).

21. MongoDB. Available online: http://www.monbgodb.com (accessed on 26 September 2016).

22. JSON. Available online: http://www.json.org (accessed on 26 September 2016).

23. XML. Available online: https://www.w3.org/XML/ (accessed on 26 September 2016).

(C) 2017 by the authors; licensee MDPI, Basel, Switzerland. This article is an open access article distributed under the terms and conditions of the Creative Commons Attribution (CC BY) license (http:/ / creativecommons.org/licenses/by/4.0/). 